\title{
Correlation of the AKT/mTOR signaling pathway with the clinicopathological features and prognosis of nasopharyngeal carcinoma
}

\author{
Yan Wang, Jie Sun, Ninghua Yao \\ Department of Radiotherapy, Affiliated Hospital of Nantong University, Nantong, China
}

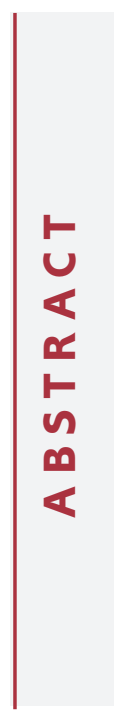

\begin{abstract}
The primary aim of this study was to examine the correlation of the AKT/mTOR signaling pathway with the clinicopathological features and prognostic significance in nasopharyngeal carcinoma (NPC). The study tissues were collected from 285 patients with NPC and normal mucosal tissues were obtained from 289 individuals with normal nasopharynxes. Immunohistochemical staining was used to detected the expression of the AKT, mTOR, and p70 ribosomal S6 kinase (P70S6K) proteins. Follow-up was performed for between 8 and 60 months. Spearman's rank correlation analysis was performed to evaluate the correlation of the expression of the AKT, mTOR, and P70S6K proteins in NPC tissues. Kaplan-Meier curves were plotted to show the survival of patients with NPC. A Cox proportional hazards model was used to explore the independent risk factors for prognosis. The expression of the AKT, mTOR, and P70S6K proteins in NPC tissues was higher than that in healthy nasopharyngeal mucosal tissues, and was correlated with T-staging, $\mathrm{N}$-staging, clinical stage, distant metastasis, and differentiation. The positive expression of the AKT, mTOR, and P70S6K proteins was higher in patients with stage III/IV NPC, low differentiation, and metastasis. The survival rates of patients with NPC with AKT-positive, mTOR-positive, and P70S6K-positive expression were considerably lower than those without the expression of these proteins. Distant metastasis and the overexpression of the AKT, mTOR, and P70S6K proteins were independent risk factors for the prognosis of patients with NPC. The results obtained from this study indicated an association between the AKT/mTOR signaling pathway and the progression of NPC. The upregulation of the AKT/mTOR pathway in patients with NPC is a predictor of poor prognosis.
\end{abstract}

Key words: AKT; mTOR; P70S6K; nasopharyngeal carcinoma; prognosis.

Correspondence: Ninghua Yao, Department of Radiotherapy, Affiliated Hospital of Nantong University, Nantong, China. E-mail: tdfywy1991@126.com

Contribution: NY, conceived the study and designed the experiments; YW, completed the experiment, analyzed the data and wrote the manuscript; JS, NY, discussed the results and revised the manuscript. All the authors have read and approved the final version of the manuscript and agreed to be accountable for all aspects of the work.

Conflict of interest: The authors declare that they have no competing interests.

Funding: This work received no funding.

Availability of data and materials: The datasets used and/or analyzed during the current study are available from the corresponding author on reasonable request.

Ethical Approval: Ethical approval was obtained for all experimental procedures by the Ethic Committee of the Affiliated Hospital of Nantong University.

Patient consent for publication: Written informed consent was obtained from the patients for their anonymized information to be published in this article. 


\section{Introduction}

As a typical carcinoma of the neck and head region, nasopharyngeal carcinoma (NPC) originates from the epithelial lining of the nasopharynx. ${ }^{1}$ There is a high incidence of NPC in Southern China and some places in Southeast Asia; every year, approximately 20-30 patients out of 100,000 will experience treatment failure owing to tumor recurrence and metastasis. ${ }^{2,3}$ Radiotherapy and other treatment regimens, including surgery, can be performed to ensure long-term local control and improve overall survival in a huge proportion of patients with NPC. ${ }^{4}$ The majority of patients who receive an early diagnosis can be completely cured if treated in the early stages (stages I and II) of the disease. ${ }^{5}$ Despite the recent rapid advancements in radiation therapy and neoadjuvant chemotherapy, patients with advanced NPC (stages III and IV) still have a poor prognosis, with a 5-year survival rate between $50 \%$ and $70 \%{ }^{6}$ It has been demonstrated that many factors are correlated with the etiology of NPC, including Epstein Barr virus (EBV) infection, environmental elements, and genetic factors. ${ }^{7}$ The longterm survival rate in patients with NPC is very low. A previous study showed an association between the AKT/mTOR signaling pathway and the prognosis of patients with NPC, and also that the high expression of the AKT/mTOR signaling pathway-related proteins affected the occurrence and development of NPC. ${ }^{8}$ Therefore, the aim of this study was to analyze the expression of the $\mathrm{AKT} / \mathrm{mTOR}$ signaling pathway in NPC and determine the correlation between the expression of these proteins and the poor prognosis of patients. The AKT/mTOR signaling pathway, which can promote the growth of tumor cells, is also highly expressed in other cancers, such as prostate cancer, gastric cancer, and ovarian cancer. The inhibition of AKT/mTOR pathway activity can effectively inhibit the growth and proliferation of tumor cells. ${ }^{9}$

AKT, a serine/threonine protein kinase, has emerged as an important regulator of the response to cellular stimuli. The inhibition of AKT was reported to suppress tumor growth. ${ }^{10}$ AKT participates in the regulation of cell proliferation, growth, and survival in many human cancers. ${ }^{11}$ Mammalian target of rapamycin (mTOR), a $289 \mathrm{kDa}$ serine/threonine kinase, serves as a regulator during the processes of angiogenesis and metastasis that affect cancer development. ${ }^{12,13}$ The activation of mTOR by AKT, mediated through the activation and phosphorylation of tuberous sclerosis complex 2 (TSC2), produces a complex that can stimulate ribosomal protein $\mathrm{S} 6$ kinase (S6K) and repress 4E binding protein (4EBP); subsequently, these enhanced protein translation. ${ }^{14}$ The $\mathrm{P} 70$ ribosomal protein S6 kinase (P70S6K) is a cytoplasmic serine/threonine kinase, and the abnormal expression of P70S6K increases ribosomal production, proliferating the translation of mRNA, which is likely to be the cause of the occurrence, development, and metastasis of gastric carcinoma. ${ }^{15}$ In addition to the above genes, there are many other highly expressed factors that promote cell growth and proliferation in NPC, such as SSX2IP and CDK4. However, further analysis is needed to determine whether the expression of these genes is related to the prognosis of NPC. ${ }^{16}$

Although NPC has been extensively investigated, reports on the effects of the AKT/mTOR signaling pathway on the clinicopathological features and prognostic significance in NPC are rare. ${ }^{17}$ Therefore, in this study, the expression of the AKT/mTOR signaling pathway and $\mathrm{P} 70$ ribosomal protein S6 kinase (P70S6K) in patients with was detected to investigate how the AKT/mTOR signaling pathway was related to the prognosis of patients with NPC.

\section{Materials and Methods}

\section{Study subjects}

Tissues were collected from 285 patients with primary NPC who underwent surgical resection in the Affiliated Hospital of Nantong University between January 2017 and July 2020. The inclusion criteria were: ${ }^{18}$ patients who were initially diagnosed with primary NPC and were not treated with any drugs, chemotherapy, or immunobiological therapy; patients with a diagnosis of nasopharyngeal squamous cell carcinoma confirmed by pathological examination, including biopsy, puncture, immunohistochemical (IHC) analysis, and imaging; complete clinical records and other related materials were available. The exclusion criteria were: ${ }^{18}$ patients with other cancers or other major diseases, including cardiovascular disease, diabetes, uremia, severe liver and kidney dysfunction, myasthenia gravis, and severe malnutrition. The included patients were 184 men and 101 women, between 18 and 71 years of age (mean age $=46.5 \pm 8.9$ years). The included subjects were classified into non-keratinized squamous cell carcinoma (SCC) (191 cases) and keratinized SCC (94 cases) in accordance with the pathological staging proposed by the World Health Organization (WHO). ${ }^{18}$ The tumor-node-metastasis (TNM) stages and clinical stages of patients with NPC were determined with reference to the $6^{\text {th }}$ edition of American Joint Committee on Cancer (AJCC) Staging System for NPC: ${ }^{19} 101$ cases of T1-T2 stage, 184 cases of T3-T4 stage; 96 cases of N0-N1 stage, 189 cases of N2N3 stage; and 94 cases of clinical stage I + II, 191 cases of stage III + IV. In addition, 289 biopsy specimens from normal nasopharynx mucosa were collected from the laryngoscope patients of Department of Otolaryngology in the Affiliated Hospital of Nantong University. The 289 specimens were taken from 177 male subjects and 112 female subjects between 15 and 63 years of age (mean age $=45.4 \pm 6.4$ years), and were confirmed as normal via pathological examination. The Ethics Committee of the Affiliated Hospital of Nantong University approved this study. Written informed consent was obtained for all study participants.

\section{Immunohistochemical staining}

Two-step PV-9000 IHC staining was conducted to examine the expression of proteins, including AKT, mTOR, and P70S6K, which is the $40 \mathrm{~S}$ sub-ribosome downstream of the AKT/mTOR signaling pathway. Tissues were blocked, dehydrated in graded ethanol solutions, permeabilized in xylene, and then embedded in paraffin. After the tissues were sliced into $4 \mu \mathrm{m}$ serial sections, deparaffinized, and hydrated using a graded ethanol solution, hightemperature antigen retrieval was performed. The slides were immersed in $3 \% \mathrm{H}_{2} \mathrm{O}_{2}$ to inactivate endogenous peroxidases, and mouse anti-human antibodies to AKT, mTOR, and P70S6K (all 1:500 dilutions) were added to the slides and incubated in a refrigerator at $4{ }^{\circ} \mathrm{C}$ overnight. Polymerase was added and incubated at room temperature for $20 \mathrm{~min}$. Horseradish peroxidase-labeled goat anti-mouse antibody (1:5000 dilution) was added to the slides and incubated at room temperature for $30 \mathrm{~min}$. Diaminobenzidine (DAB) was added as a chromogenic agent, and hematoxylin was added for redyeing and mounting. In the normal nasopharynx mucosal tissues, which were considered as the control, positive staining of AKT, mTOR, and P70S6K proteins was observed in the cytoplasm with light yellow, brown-yellow, or brown particles. The positive expression of proteins referred to the occurrence of brown-yellow particles in the nuclei. Five randomly selected fields for each stained section were analyzed at high power $(\times 200)$. The percentage of positively stained cells in the total cell count was calculated as the labeling index (LI) of the proteins. An LI of $>10 \%$ indicated positive expression of the protein and an LI of $\leq 10 \%$ 
indicated that the protein was not expressed. ${ }^{20}$ Two experienced pathologists independently evaluated all the experimental results in a double-blinded manner.

\section{Follow-up and prognosis}

The follow-up of 285 patients started from the date of confirmed diagnosis and ended in July 2016. The means of follow-up included reviewing the records associated with return visits and performing telephone surveys. The endpoint of this study was overall survival (OS), and was defined as the period of time between the date of initial diagnosis and the date of death or the date of last visit. The follow-up lasted for between 8 and 60 months; the mean follow-up period was 43.7 months. Of the 285 included cases, complete follow-up data were obtained for 261 cases; 24 cases were lost to follow-up. The rate of effective followup was $91.58 \%$, which was more intensive follow-up.

\section{Statistical analysis}

All statistical analyses were conducted using SPSS 21.0 software. The experimental data were expressed as the mean \pm standard deviation (SD). The $t$-test was used for the comparison between two groups and the analysis of variance was used for multiple pairwise comparisons. Percentage or rate were used to express numerical data and the pairwise comparison was examined by the chi-square test. Spearman's rank correlation analysis was employed to assess correlations. The Kaplan-Meier curve was plotted to show the survival of patients, and the pairwise comparison was evaluated by a log-rank test. To determine the independent prognostic factors related to OS of patients with NPC, a Cox proportional hazards model was conducted. All experiments were repeated three times; $p$-values of $<0.05$ were considered statistically significant.

\section{Results}

\section{Baseline characteristics of patients with NPC and nor- mal controls.}

The NPC group included 184 men and 101 women with a mean age of $46.5 \pm 8.9$ years of age. The normal control group included 177 men and 112 women with a mean age of $45.6 \pm 5.8$ years. No statistically significant differences between the two groups were found for age, sex, smoking history, or alcohol use (all $\mathrm{p}>0$. 05) (Table 1).

\section{Immunohistochemical analysis of AKT, mTOR, and P70S6K in NPC tissues and normal nasopharyngeal mucosal tissues}

The positive expression of AKT, mTOR, and P70S6K was identified in the cytoplasm by light yellow, brown-yellow, or brown particles (Figure 1). The positive expression rates of AKT,

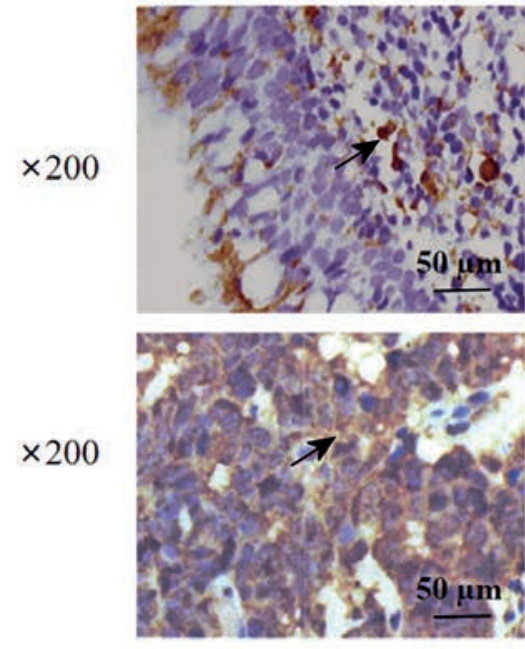

AKT
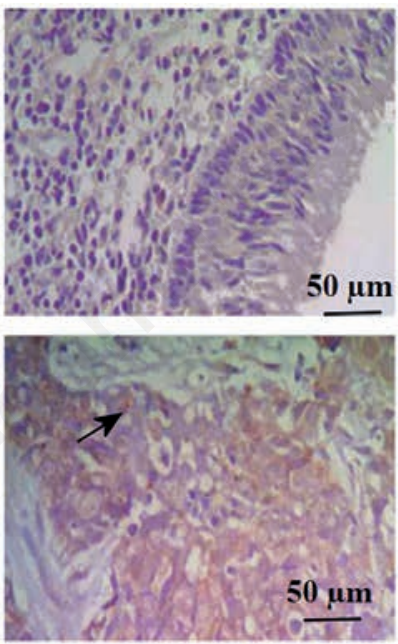

mTOR

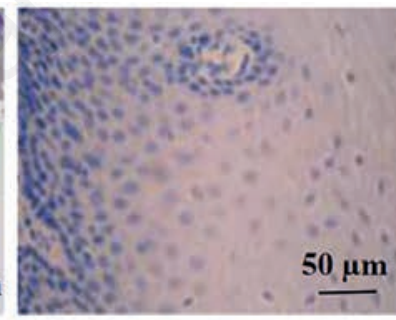

Normal Tissue

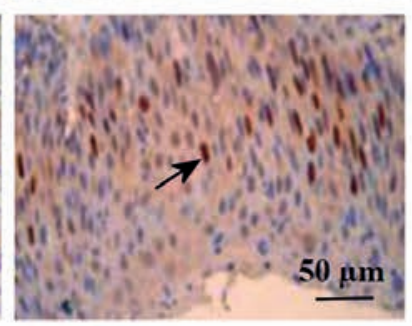

Nasopharyngeal

Carcinoma

Figure 1. Expressions of AKT, mTOR and P70S6K protein in NPC tissues and normal mucosal tissues $(\times 200)$. The positive staining of AKT, mTOR and P70S6K were located in the cytoplasm with light yellow, brown-yellow or brown particles; mTOR, mammalian target of rapamycin; P70S6K, p70 ribosomal S6 kinase.

Table 1. Baseline characteristics of the NPC group and the normal control group.

\begin{tabular}{|c|c|c|c|c|}
\hline Factor & NPC group ( $n=285)$ & Normal control group $(\mathrm{n}=289)$ & $t / \chi^{2}$ & p \\
\hline Mean age & $46.5 \pm 8.9$ & $45.6 \pm 6.4$ & 1.702 & 0.089 \\
\hline Gender (male/female) & $184 / 101$ & $177 / 112$ & 0.676 & 0.411 \\
\hline Smoking history (yes/no) & $169 / 123$ & $174 / 115$ & 0.049 & 0.824 \\
\hline Alcohol use (yes/no) & $120 / 165$ & $114 / 175$ & 0.420 & 0.517 \\
\hline
\end{tabular}

NPC, nasopharyngeal carcinoma. 
mTOR, and P70S6K in NPC tissues were 68.77\% (196/285), $74.39 \%(212 / 285)$, and $60.00 \%(171 / 285)$ respectively. The positive expression rates of AKT, mTOR, and P70S6K in normal nasopharyngeal mucosal tissues were $38.41 \%$ (111/289), 36.68\% $(106 / 289)$ and $25.61 \%(74 / 289)$. The expression of the AKT, mTOR, and P70S6K proteins in NPC tissues was higher than that in normal nasopharyngeal mucosal tissues (all $\mathrm{p}<0.05$ ) (Figure 2).

Correlations of protein expression of AKT, mTOR, and P70S6K with the clinicopathological characteristics of patients with NPC

The correlations of the expression of the AKT, mTOR, and P70S6K proteins with clinicopathological characteristics of patients with NPC are shown in Table 2. The expressions of the AKT, mTOR, and P70S6K proteins were correlated with T-staging, $\mathrm{N}$-staging, clinical stages, distant metastasis, and differentiation (all $\mathrm{p}<0.05$ ). Positive expression of the AKT, mTOR, and P70S6K proteins was higher in patients with stage III/IV NPC, low differentiation, and metastasis. There were no correlations between the expression of the AKT, mTOR, and P70S6K proteins and age, sex, smoking history, alcohol use, and pathological stage in all patients $(\mathrm{p}>0.05)$

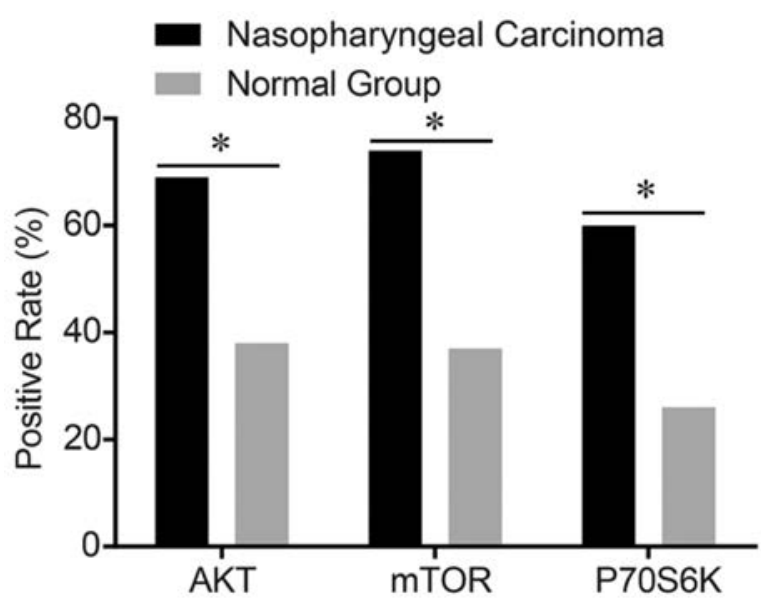

Figure 2. Comparisons on positive expressions of AKT, mTOR and P70S6K proteins in the NPC group and the normal control group. ${ }^{*} \mathbf{p}<\mathbf{0 . 0 5}$ compared with the normal control group; NPC, nasopharyngeal carcinoma; mTOR, mammalian target of rapamycin; P70S6K, p70 ribosomal S6 kinase.

Table 2. Correlations of expressions of AKT, mTOR and P70S6K with clinicopathological features of patients with NPC.

\begin{tabular}{|c|c|c|c|c|c|c|c|}
\hline Clinicopathological feature & n & $\begin{array}{l}\text { Positive } \\
\text { expression of } \\
\text { AKT (\%) }\end{array}$ & p & $\begin{array}{l}\text { Positive } \\
\text { expression of } \\
\text { mTOR }(\%)\end{array}$ & p & $\begin{array}{l}\text { Positive } \\
\text { expression of } \\
\text { P70S6K (\%) }\end{array}$ & p \\
\hline $\begin{array}{l}\text { Age (year) } \\
\quad<50 \\
\quad \geq 50\end{array}$ & $\begin{array}{l}181 \\
104\end{array}$ & $\begin{array}{l}70.72 \% \\
65.38 \%\end{array}$ & 0.356 & $\begin{array}{l}74.03 \% \\
75.00 \%\end{array}$ & 0.889 & $\begin{array}{l}58.56 \% \\
62.50 \%\end{array}$ & 0.533 \\
\hline $\begin{array}{l}\text { Gender } \\
\text { Male } \\
\text { Female }\end{array}$ & $\begin{array}{l}184 \\
101 \\
\end{array}$ & $\begin{array}{l}67.39 \% \\
71.29 \% \\
\end{array}$ & 0.593 & $\begin{array}{l}73.37 \% \\
76.24 \%\end{array}$ & 0.671 & $\begin{array}{l}61.96 \% \\
56.44 \%\end{array}$ & 0.379 \\
\hline $\begin{array}{l}\text { Smoking history } \\
\text { Yes } \\
\text { No }\end{array}$ & $\begin{array}{l}162 \\
123\end{array}$ & $\begin{array}{l}66.05 \% \\
72.36 \%\end{array}$ & 0.302 & $\begin{array}{l}72.22 \% \\
77.24 \%\end{array}$ & 0.411 & $\begin{array}{l}58.02 \% \\
62.60 \%\end{array}$ & 0.465 \\
\hline $\begin{array}{l}\text { Alcohol use } \\
\text { Yes } \\
\text { No }\end{array}$ & $\begin{array}{l}120 \\
165\end{array}$ & $\begin{array}{l}70.83 \% \\
67.27 \%\end{array}$ & 0.605 & $\begin{array}{l}75.00 \% \\
73.94 \%\end{array}$ & 0.891 & $\begin{array}{l}60.83 \% \\
59.39 \%\end{array}$ & 0.903 \\
\hline $\begin{array}{l}\text { Pathological stages } \\
\text { Keratinizing } \\
\text { Non-keratinizing }\end{array}$ & $\begin{array}{c}94 \\
191\end{array}$ & $\begin{array}{l}71.28 \% \\
67.54 \%\end{array}$ & 0.587 & $\begin{array}{l}79.79 \% \\
71.73 \%\end{array}$ & 0.152 & $\begin{array}{l}65.96 \% \\
57.07 \%\end{array}$ & 0.159 \\
\hline $\begin{array}{r}\text { T-staging } \\
\text { T1-T2 } \\
\text { T3-T4 }\end{array}$ & $\begin{array}{l}101 \\
184\end{array}$ & $\begin{array}{l}55.45 \% \\
76.09 \%\end{array}$ & 0.001 & $\begin{array}{l}51.49 \% \\
86.96 \%\end{array}$ & $<0.001$ & $\begin{array}{l}35.64 \% \\
73.37 \%\end{array}$ & $<0.001$ \\
\hline $\begin{array}{r}\text { N-staging } \\
\text { N0-N1 } \\
\text { N2-N3 }\end{array}$ & $\begin{array}{c}96 \\
189\end{array}$ & $\begin{array}{l}60.42 \% \\
73.02 \%\end{array}$ & 0.042 & $\begin{array}{l}53.13 \% \\
85.19 \%\end{array}$ & $<0.001$ & $\begin{array}{l}37.50 \% \\
71.43 \%\end{array}$ & $<0.001$ \\
\hline $\begin{array}{l}\text { Distant metastasis } \\
\text { No } \\
\text { Yes }\end{array}$ & $\begin{array}{c}79 \\
205\end{array}$ & $\begin{array}{l}53.16 \% \\
74.63 \%\end{array}$ & 0.001 & $\begin{array}{l}51.90 \% \\
83.41 \%\end{array}$ & $<0.001$ & $\begin{array}{l}39.24 \% \\
68.29 \%\end{array}$ & $<0.001$ \\
\hline $\begin{array}{l}\text { Clinical staging } \\
\text { I/II stage } \\
\text { III/IV stage }\end{array}$ & $\begin{array}{c}94 \\
191\end{array}$ & $\begin{array}{l}60.64 \% \\
72.77 \%\end{array}$ & 0.042 & $\begin{array}{l}55.32 \% \\
83.77 \%\end{array}$ & $<0.001$ & $\begin{array}{l}41.49 \% \\
69.11 \%\end{array}$ & $<0.001$ \\
\hline $\begin{array}{l}\text { Differentiation degree } \\
\text { Low/undifferentiation } \\
\text { Medium/high differentiation }\end{array}$ & $\begin{array}{c}218 \\
67\end{array}$ & $\begin{array}{l}76.15 \% \\
44.78 \%\end{array}$ & $<0.001$ & $\begin{array}{l}83.03 \% \\
46.27 \%\end{array}$ & $<0.001$ & $\begin{array}{l}67.89 \% \\
34.33 \%\end{array}$ & $<0.001$ \\
\hline
\end{tabular}

mTOR, mammalian target of rapamycin; P70S6K, p70 ribosomal S6 kinase; NPC, nasopharyngeal carcinoma. 
Correlations of expression of the AKT, mTOR, and P70S6K proteins in NPC

According to Spearman's correlation analysis (Table 3), the positive expression of the AKT and mTOR proteins were positively correlated in patients with NPC $(\mathrm{r}=0.455, \mathrm{p}<0.001)$, and there was a low but not significant positive correlation of AKT with P70S6K in NPC ( $r=0.037, p=0.553)$. The positive expression of P70S6K was positively correlated with the expression of mTOR ( $r$ $=0.456, \mathrm{p}<0.001)$.

Correlation of expression of the AKT, mTOR, and P70S6K proteins with survival rates of patients with NPC

The expression of the AKT, mTOR, and P70S6K proteins in NPC was analyzed by dividing the patients into a negative-expression group and a positive-expression using immunohistochemical staining. The Kaplan-Meier survival curves of AKT, mTOR, and P70S6K proteins were displayed in Figure 3. The survival rate of patients with positive expressions of the AKT, mTOR, and P70S6K proteins was significantly lower than that of patients in the negative-expression group (all $\mathrm{p}<0.05$ ).

Multivariate analysis of the risk factors for prognosis in patients with NPC using the Cox proportional hazards model

The Cox proportional hazards model (Table 4) was used to evaluate whether the expression of the AKT, mTOR, and P70S6K proteins and clinicopathological features were independent risk factors for patients with NPC. The results showed that age, T-staging, $\mathrm{N}$-staging, and clinical stage were not independent risk factors (all $\mathrm{p}>0.05$ ). Distant metastasis and the overexpression of the AKT, mTOR, and P70S6K proteins were independent risk factors for the prognosis of patients with NPC (all $\mathrm{p}<0.05)$.

\section{Discussion}

NPC is biologically different from other head and neck cancers, and early diagnosis is difficult because the symptoms are not obvious. In addition, NPC is usually treated conservatively owing to the difficulty and complexity of nasopharyngeal surgery and the mechanics of NPC are still unclear..$^{21,22}$ Therefore, there is an urgent need for new research into the treatment of nasopharyngeal cancer. The AKT/mTOR signaling pathway plays an essential role in mediating cellular processes, such as cell growth, cellular proliferation, cell differentiation, and survival. ${ }^{23,24}$ The precise underlying mechanism of the roles of AKT and mTOR in NPC should

Table 3. Correlations of expressions of AKT, mTOR and P70S6K in NPC tissues.

\begin{tabular}{lccc} 
Protein & AKT & m'lOR & P70S6K \\
AKT & 1 & $0.455^{\mathrm{a}}$ & 0.037 \\
mTOR & $0.455^{\mathrm{a}}$ & 1 & $0.456^{\mathrm{a}}$ \\
P70S6K & 0.037 & $0.456 \mathrm{a}$ & 1 \\
\hline
\end{tabular}

Note: a, $\mathrm{P}<0.0001$; mTOR, mammalian target of rapamycin; P70S6K, p70 ribosomal S6 kinase; NPC, nasopharyngeal carcinoma

Table 4. Multivariate analysis with Cox proportional hazards model on risk factors for prognosis of NPC patients.

\begin{tabular}{|c|c|c|c|c|c|c|}
\hline Factor & SE & Wald & p & $\operatorname{EXP}(B)$ & & \\
\hline & & & & & Upper limit & Lower limit \\
\hline Age & 0.145 & 0.014 & 0.905 & 1.017 & 0.765 & 1.353 \\
\hline Gender & 0.146 & 0.416 & 0.519 & 1.099 & 0.826 & 1.462 \\
\hline Pathological staging & 0.148 & 0.143 & 0.705 & 0.945 & 0.707 & 1.264 \\
\hline T-staging & 0.189 & 3.276 & 0.070 & 1.408 & 0.972 & 2.039 \\
\hline N-staging & 0.184 & 0.111 & 0.740 & 1.063 & 0.742 & 1.523 \\
\hline Clinical staging & 0.182 & 0.148 & 0.700 & 1.073 & 0.751 & 1.533 \\
\hline Distant metastasis & 0.069 & 4.087 & 0.043 & 1.150 & 1.004 & 1.316 \\
\hline AKT expression & 0.185 & 5.499 & 0.019 & 1.543 & 1.074 & 2.217 \\
\hline mTOR expression & 0.261 & 10.907 & 0.001 & 2.365 & 1.419 & 3.943 \\
\hline P70S6K expression & 0.178 & 7.610 & 0.006 & 1.635 & 1.153 & 2.318 \\
\hline
\end{tabular}

NPC, nasopharyngeal carcinoma; mTOR, mammalian target of rapamycin; P70S6K, p70 ribosomal S6 kinase; SE, standard error; CI, confidence interval.

A

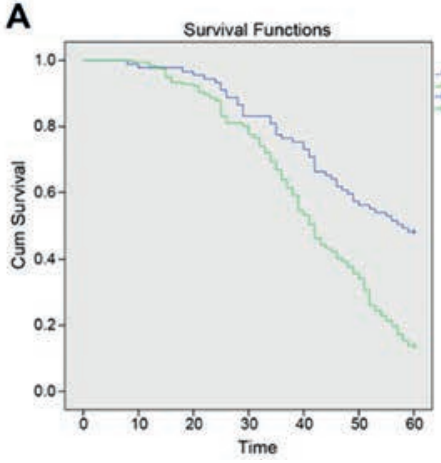

B

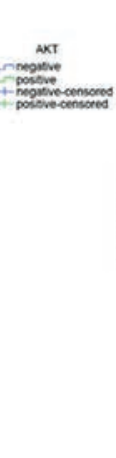

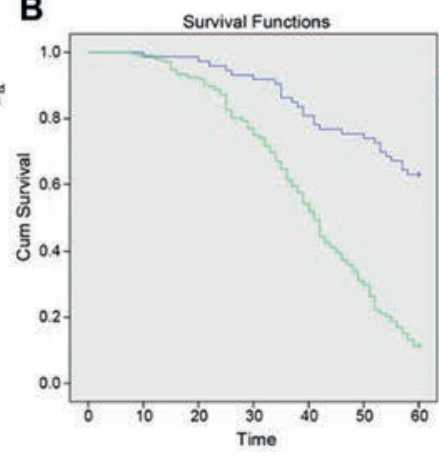

c

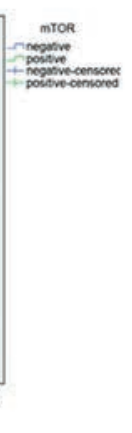

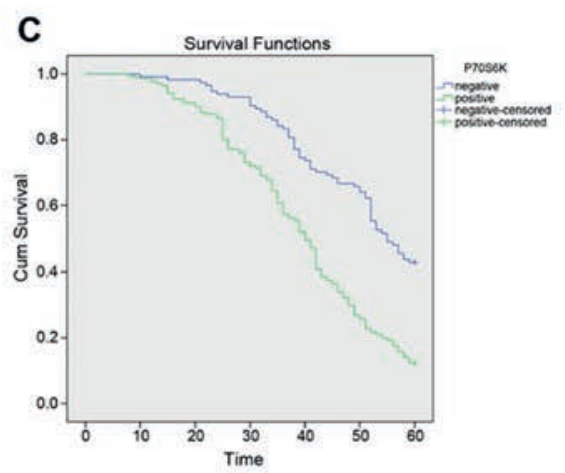

Figure 3. Kaplan-Meier curves of survival rates of NPC patients with different expressions of AKT, mTOR and P70S6K. A, expression of AKT protein; B, expression of mTOR protein; C, expression of P70S6K protein; NPC, nasopharyngeal carcinoma. 
be determined, as an association with targeted therapy in cancer treatment has been shown. ${ }^{25,26}$ As shown in our study, the AKT/mTOR signaling pathway may be related to the development and progression of NPC; in addition, the overexpression of the AKT, mTOR, and P70S6K proteins was indicative of poor NPC prognosis. The experimental results of the present study showed that positive expression of the AKT, mTOR, and P70S6K proteins was increased in NPC tissue, with greater increases in patients with stage III/IV NPC, low differentiation, and metastasis. Moreover, this study demonstrated the close associations of AKT, mTOR, and P70S6K with NPC in T-staging, N-staging, clinical stages, distant metastasis, and differentiation. There is evidence for the abnormal expression of the AKT/mTOR signaling pathway in multiple malignant tumors, including bladder cancer, colon cancer, and prostate cancer. ${ }^{27-29}$ In prostate cancer, the inhibition of the AKT/mTOR signaling pathway can enhance radiosensitivity, whereas the inhibition of the AKT/mTOR signaling pathway also inhibited the proliferation, invasion, and metastasis of colon cancer cells and reduced the number of invasive lesions in of bladder cancer. Our results suggest that the AKT/mTOR signaling pathway may play a role in NPC, similar to its effects in colon, prostate, and bladder cancers. AKT regulates downstream signaling through the phosphorylation of substrate proteins to mediate multiple phenotypes and is correlated with features of malignancy, such as cell growth, apoptosis evasion, aggressive migration, angiogenesis, and metabolic reprogramming. ${ }^{30}$ As shown in a previous study, the mechanism of radioresistance in multiple human cancers, such as neck and head cancer (HNC), and brain cancer, is related to the activation of the AKT/mTOR signaling pathway, ${ }^{31,32} \mathrm{AKT}, \mathrm{mTOR}$, and 4E-binding protein 1 (4EBP1). Owing to its correlation with AKT, the mTOR signaling pathway is phosphorylated and activated, but the AKT/mTOR signaling pathway-related proteins are expressed abnormally, which leads to NPC progression. ${ }^{33}$ A recent study showed that the PI3K/AKT/mTOR signaling pathway with a high expression of the AKT and mTOR proteins was regarded as a critical factor for predicting the unfavorable prognosis of NPC. ${ }^{34}$ P70S6K and 4EBP1, two components of the mTOR signaling pathway, as well as the regulators of mTOR signaling pathway growth, are phosphorylated by latent membrane protein-1 (LMP1), which modulates the pathological development of NPC, including cell apoptosis, cellular proliferation, and metastasis..$^{35}$

Our study indicated that patients with NPC and positive expression of the P70S6K protein may have a lower survival probability than patients with NPC and no expression of the P70S6K protein. In the study of Zhang et al., P70S6K was shown to exert crucial roles in maintaining the proliferation and survival of NPC cells, and the PI3K/AKT signaling blockade may inhibit the proliferation and enhance apoptosis in NPC cells. ${ }^{36}$ In terms of 4EBP1 and P70S6K, 4EBP1 participates in and also regulates the synthesis of ribosomal proteins, and non-phosphorylated 4EP1 promotes the initiation complex, translation inhibition, cell apoptosis, and represses the survival and proliferation of tumor cells. ${ }^{37}$ Evidence on a similar topic has been provided, showing that the activation of the mTOR protein by phosphorylation and the expression of ribosomal protein $\mathrm{S} 6$ had a negative impact on the survival of patients with NPC. ${ }^{38}$ According to the Cox proportional hazards model, the overexpression of the AKT, mTOR, and P70S6K proteins resulted in the poor prognosis of patients with NPC; consequently, this finding may propose new methods for both prognostic prediction and novel targeted therapy. It was shown in previous studies that mTOR could induce the activation of downstream factors and promote the malignancy of NPC, and that a high expression of mTOR was related to the negative prognosis of patients with NPC. ${ }^{39}$ The activation and overexpression of P70S6K enhanced metastasis and poor prognosis in HNC through the induction of the epithelial- mesenchymal transition (EMT). ${ }^{40}$ The effects of P70S6K on cancer metastasis in the mTOR signaling pathway have been determined previously, and activation of the mTOR signaling pathway was shown to potentiate the EMT, invasion, migration, and metastasis. ${ }^{41,42}$

The patients with NPC included in this study received only limited follow-up, and there were more male patients with NPC than female patients. Owing to the limitations on our research, we did not attempt to determine the expression of the related genes in this study, and our comparisons are not sufficiently comprehensive. All of these factors may affect the experimental results. Thus, further studies, with a longer follow-up period, an equal ratio of male and female subjects, and further studies, are needed to confirm these results. However, this study indicated that there was a correlation between the AKT/mTOR signaling pathway and the development and progression of NPC, and that unfavorable prognosis may result from the overexpression of the AKT, mTOR, and P70S6K proteins. Moreover, it is suggested that the AKT/mTOR signaling pathway may act as a prospective prognostic predictor and novel therapeutic target. There are many genes that may be related to the prognosis of patients with NPC, and these can be studied further to provide more effective therapeutic targets and markers for the early diagnosis of NPC.

\section{References}

1. Yang L, Tang Y, He Y, Wang Y, Lian Y, Xiong F, et al. High expression of LINC01420 indicates an unfavorable prognosis and modulates cell migration and invasion in nasopharyngeal carcinoma. J Cancer 2017;8:97-103.

2. Xu L, Fan S, Zhao J, Zhou P, Chu S, Luo J, et al. Increased expression of Cks1 protein is associated with lymph node metastasis and poor prognosis in nasopharyngeal carcinoma. Diagn Pathol 2017;12:2.

3. Wei GB, Lu YY, Liao RW, Chen QS, Zhang KQ. Prognostic nutritional index predicts prognosis in patients with metastatic nasopharyngeal carcinoma. Onco Targets Ther 2016;9:5955-61.

4. Suarez C, Rodrigo JP, Rinaldo A, Langendijk JA, Shaha AR, Ferlito A. Current treatment options for recurrent nasopharyngeal cancer. Eur Arch Otorhinolaryngol 2010;267:1811-24.

5. Chen J, Hu CF, Hou JH, Shao Q, Yan LX, Zhu XF, et al. EpsteinBarr virus encoded latent membrane protein 1 regulates mTOR signaling pathway genes which predict poor prognosis of nasopharyngeal carcinoma. J Transl Med 2010;8:30.

6. Zhou XM, Sun R, Luo DH, Sun J, Zhang MY, Wang MH, et al. Upregulated TRIM29 promotes proliferation and metastasis of nasopharyngeal carcinoma via PTEN/AKT/mTOR signal pathway. Oncotarget 2016;7:13634-50.

7. Lung ML, Cheung AK, Ko JM, Lung HL, Cheng Y, Dai W. The interplay of host genetic factors and Epstein-Barr virus in the development of nasopharyngeal carcinoma. Chin J Cancer 2014;33:556-68.

8. Wang W, Wen Q, Xu L, Xie G, Li J, Luo J, et al. Activation of $\mathrm{Akt} / \mathrm{mTOR}$ pathway is associated with poor prognosis of nasopharyngeal carcinoma. PLoS One 2014;9:e106098.

9. Wang KF, Yang H, Jiang WQ, Li S, Cai YC. Puquitinib mesylate (XC-302) induces autophagy via inhibiting the PI3K/AKT/mTOR signaling pathway in nasopharyngeal cancer cells. Int J Mol Med 2015;36:1556-62.

10. Jiang Z, Liu Y, Wang C. Oncogenic NanogP8 expression regulates cell proliferation and migration through the Akt/mTOR signaling pathway in human gastric cancer - SGC-7901cell line. Onco Targets Ther 2016;9:4859-66.

11. Feng S, Jin Y, Cui M, Zheng J. Lysine-specific demethylase 1 (LSD1) inhibitor S2101 induces autophagy via the AKT/mTOR 